\title{
Cloud Computing Environment
}

\author{
Harshita Doad $^{1}$, Dr. Trilok Gupta ${ }^{2}$ \\ Research Scholar, Career Point University, kota, Rajasthan, India. ${ }^{1}$ \\ Assistant Professor (CS) MIMT, Dadabari, Kota, Rajasthan, India. ${ }^{2}$
}

\begin{abstract}
Cloud computing is an on demand availability of cloud system resources such as data storage and computing power without direct active management by the user. The word cloud is used as a metaphor for internet and a standardized cloud like shape was used to denote a network on schematic's. [1] Cloud is about where you compute, not how you compute. Here, we will discuss about difference between and On-premises and Cloud-based computing, Scalability, server storage with cloud service providers, data security, maintenance in terms of infrastructure as well as cost efficiency. It is described as the delivery of on-demand services over the internet works on "pay-as-you-go" basis. Cloud computing has two type of models:
\end{abstract}

\section{- deployment model:}

public cloud

private cloud

hybrid cloud

\section{- $\quad$ Service model:}

infrastructure as a service

platform as a service

software as a service

Cloud computing service models are hassle-free. The most popular cloud computing models AWS, Microsoft's azure, Google cloud platform are as discussed below. SLAs, instances, container, Kubernetes, Regions, storage are all important factors also discussed in this paper. How cloud providers provide services over the cloud and what are the importance of those services in the modern day infrastructure. The data usage has been discussed providing a large dependency in processing the programs. The future introduction is also mentioned giving new research aspects to the field of cloud computing.

Keyword: Explanation of various types of cloud services provided, types of cloud being discussed, market value regarding cloud computing is mentioned, various cloud providers as well as organizations running on cloud is explained, service model is discussed with various storage properties, scalability, containers and kubernetes are briefly explained, Future of cloud is being discussed.

\section{INTRODUCTION:}

"Cloud is about how you do computing, not where you do computing." This well-known phrase is typically depicted by Paul Moritz, CEO of VMware. Cloud computing acts like a power plant, in which we don't know how the power is generated, we are just plugging into the systems and using that power and paying bills., this could also be an example of Electricity-as-a-service (Eaas).In the year 2006, Amazon Web Services launched life changing scenario: EC2 (Elastic compute cloud) and S3 (simple storage services), EC2 , acting as a virtual system with its own CPU as well as RAM, the operating system is also present which could be administered by the user with IP address available for further networking. 
Vol. 10, Issue 9, September 2021

DOI: $10.17148 /$ IJARCCE.2021.10907

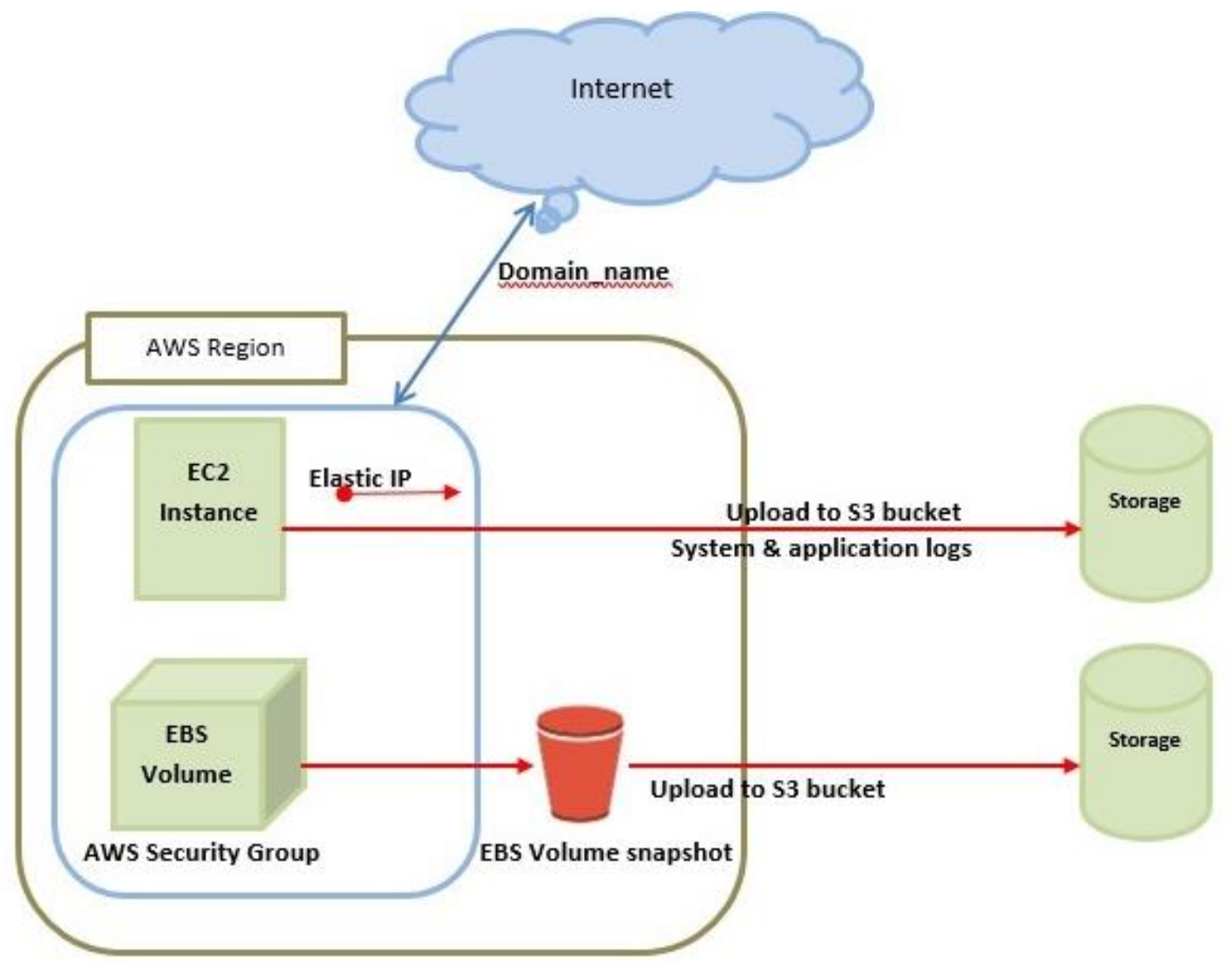

Basic architecture of the services provides over the cloud in AWS [2]

In the same year,2006, Amazon launched Simple Storage Services, this is the file system (images, videos) which is stored in the cloud in the form of hard disk.

Virtual machine could be defined as the processing server over the cloud, as one doesn't have any specific hardware attached to this system, in turn, a virtual environment is maintained which could be stated as hardware of a virtual system. The user is unaware of the fact of what goes behind the scenes, utilizing the front-end of the system. In this virtual system the hypervisor relies on the hardware connecting to various operating systems which handles CPU, scheduling and different memory allocation.

[3] Hypervisor are divided into two categories

- $\quad$ Type-1 hypervisor (bare metal): These kind of hypervisor are generally acts as operating systems running over different host hardware's,

- $\quad$ Type-II hypervisor (hosted): This appears as a software layer over an operating system similar like other computer programs. 

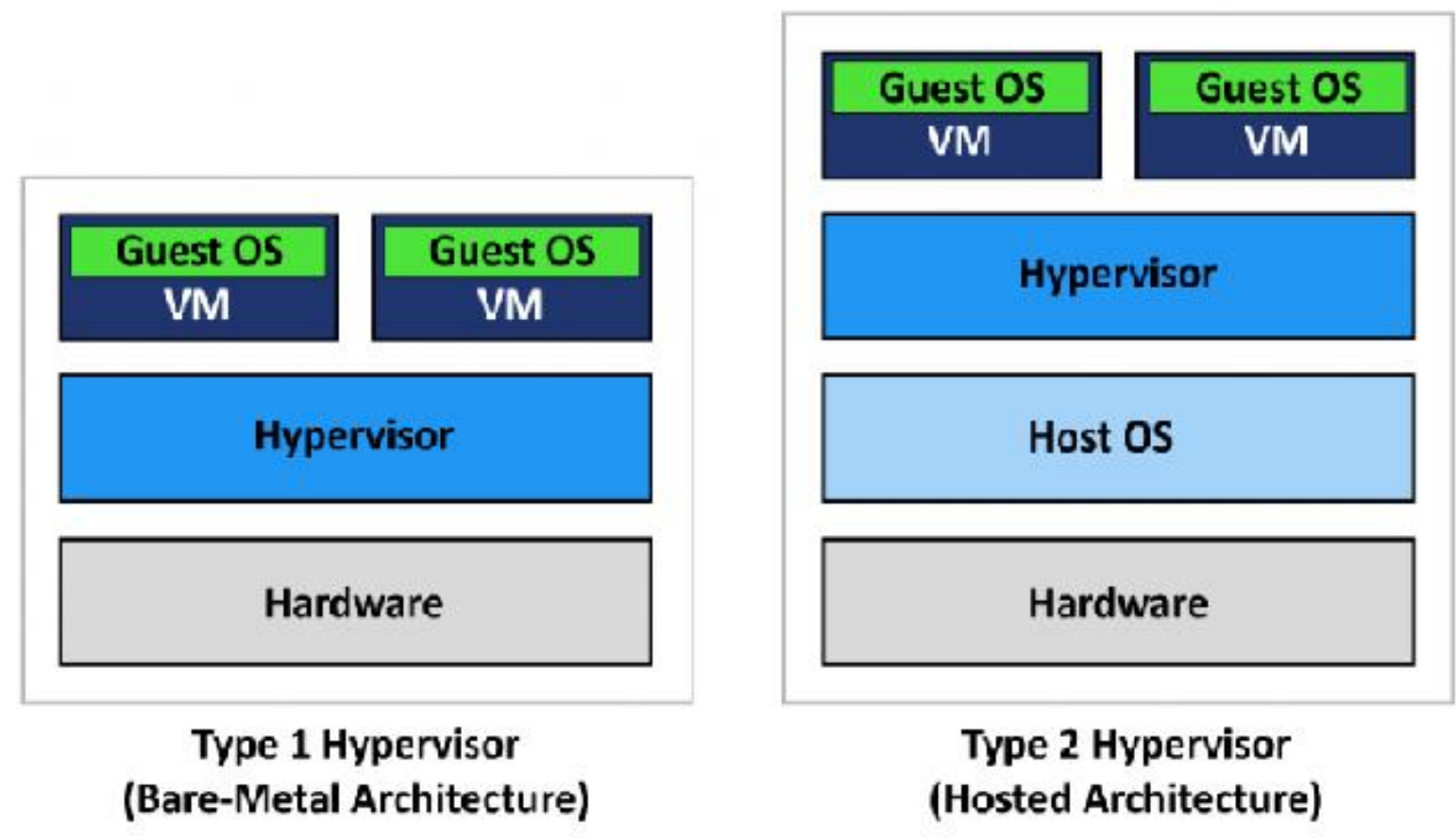

Hypervisor and Virtual box [4]

\section{METHODS:}

To describe cloud computing models for further knowledge is it has three basic services:

\section{- Infrastructure-as-a-service}

If you talk about infrastructure as a service, Virtual machines and storage buckets are the biggest examples of this kind of services. This is the lowest level of building blocks of cloud. It relies completely on the developer for management and scaling purposes. It relies heavily on self-servicing mode. Once the infrastructure-as-a service is landed in market it provided an upgradation in software-as-a-service model.

\section{- Software-as-a-service}

Software as a service that described as a new type of software Developed due to updated infrastructure. Suppose if we look upon a "Dropbox" [5], in earlier days this company doesn't have the cost required for owning its own infrastructure, hence it starts uploading its users file on the Simple Storage Service S3. This S3 was actually not a files storage company but it was a software company, used for storing various files over internet for getting the different files uploaded to the cloud Environment. This is a live example of building \$1 billion company with just focusing on user-friendly front-end system.

\section{- $\quad$ Platform-as-a-service [6]}

Infrastructure-as-a-service, totally abstract the services as the hardware system. Within few years, the cloud computing produced a new level of platform-as-a-service, any application needs to be developed not only require the hardware but also needed to take a charge for security, management as well as putting everything in an aligned manner and scalability towards the workload. Elastic beanstalk, HEROKU, Google app engine are few examples of Paas.

Suppose an app is built by a developer in order to get used by the people of providing services. one needs to have a database, specifically configured web servers, security and so on. Hence, the platform takes the charge of configuring the system, with cloud taking the charge on database, security as well as scaling the traffic. The only thing a developer needs to do is to upload the code on the system. This is actually platform-as-a-service. 
Vol. 10, Issue 9, September 2021

DOI: $10.17148 /$ IJARCCE.2021.10907

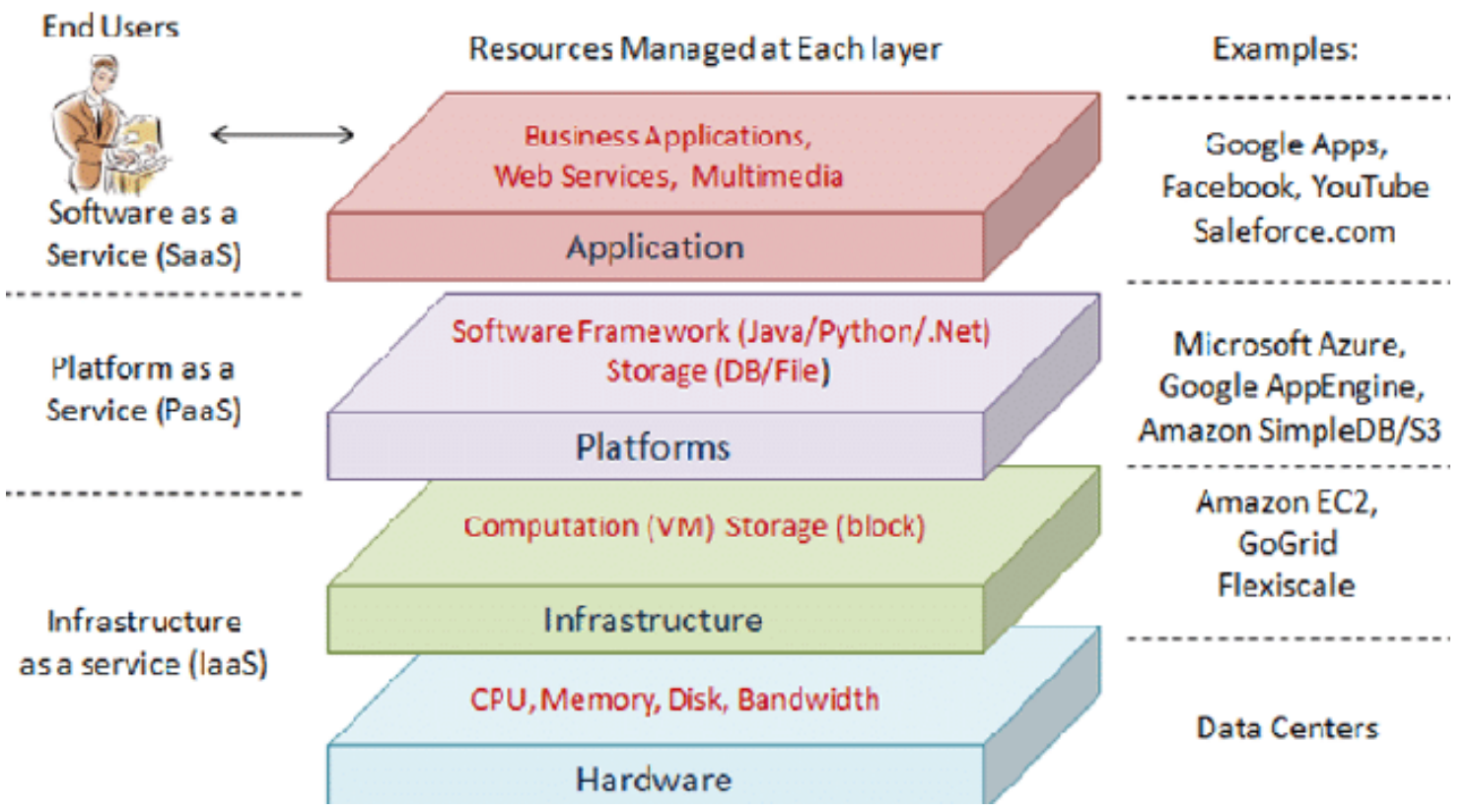

Working of cloud computing architecture at various service models. [7]

\section{- $\quad$ Backend-as-a-service}

If we go one step beyond, we also have learned about backend-as-a-service (Baas) [8], it brings the cloud directly to the applications running on front-end thus not needing any backend code which is usually generated for processing any application. It has been taken care of by Baas. Two of the main examples widely used under this system is Firebase (from google), Amplify (from AWS). A real-time application is created with the help of JavaScript, focusing on authentic users who are connected to the database of the cloud.

With all this usages, comes a little cost. There are pretty challenges for the usage of cloud computing, the more the user relied on the services provided by the cloud, the more are the chances of getting hooked to vendor lock-in. Cloud can help user in many ways, leading to the new dimensions of success but it is seen that once those dimensions are achieved, the ideas of user start changing drastically.

\section{There are various types of cloud in the environment. [9]}

\section{- $\quad$ Public cloud}

The public cloud mentioned above is the one which can be easily accessed through sign-in's and mail IDs. One of the examples of public now are AWS, Azure, GCP.

\section{- $\quad$ Private cloud}

With the private cloud the cloud infrastructure is specifically operated on by a single organization or a third party. It is used by the larger companies who have the ability of purchasing their own cloud. Companies such as AWS, Azure, Google, IBM comes under this type of cloud.

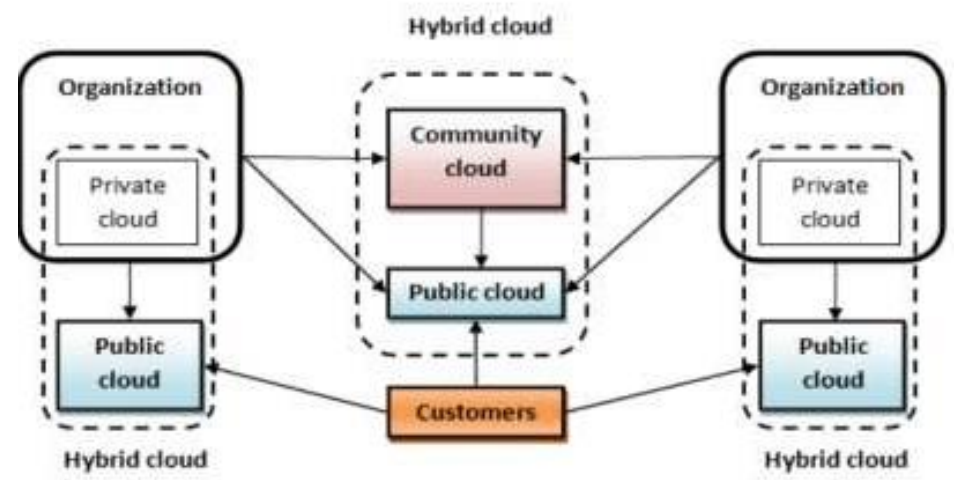

Cloud computing deployment model [10] 
Vol. 10, Issue 9, September 2021

DOI: $10.17148 / I J A R C C E .2021 .10907$

\section{- $\quad$ Hybrid cloud}

In 2016, the same company "dropbox" started to move the data and files to their own data centers from storage systems S3, saving almost $\$ 75$ million from their operating expenses. Thus, in this case, moving the system to an on-premises location from the cloud was a great decision.

Although the system didn't go completely on-premises. It still has a little existence on the cloud by uploading various files of the regions which the data centers are unable to cover. Now this kind of system is an example of hybrid cloud (on-premises + public cloud), It basically used for the larger organizations which have their own data center cloud as well as they could purchase services from the public cloud.

\section{- $\quad$ Multi cloud [11]}

The multi cloud refers to the architecture or the applications which combines various services from different public clouds. They are highly used to prevent vendor login and optimize pricing. This is basically a mix and match of different clouds.

Service level agreements SLA, they provide an agreement between user and the cloud provider. The provider provides with certain up-time as well as error rate for the services and also gives the benefit of providing a refund or financial credit bank, if the provider fails to meet his stated SLA requirements.

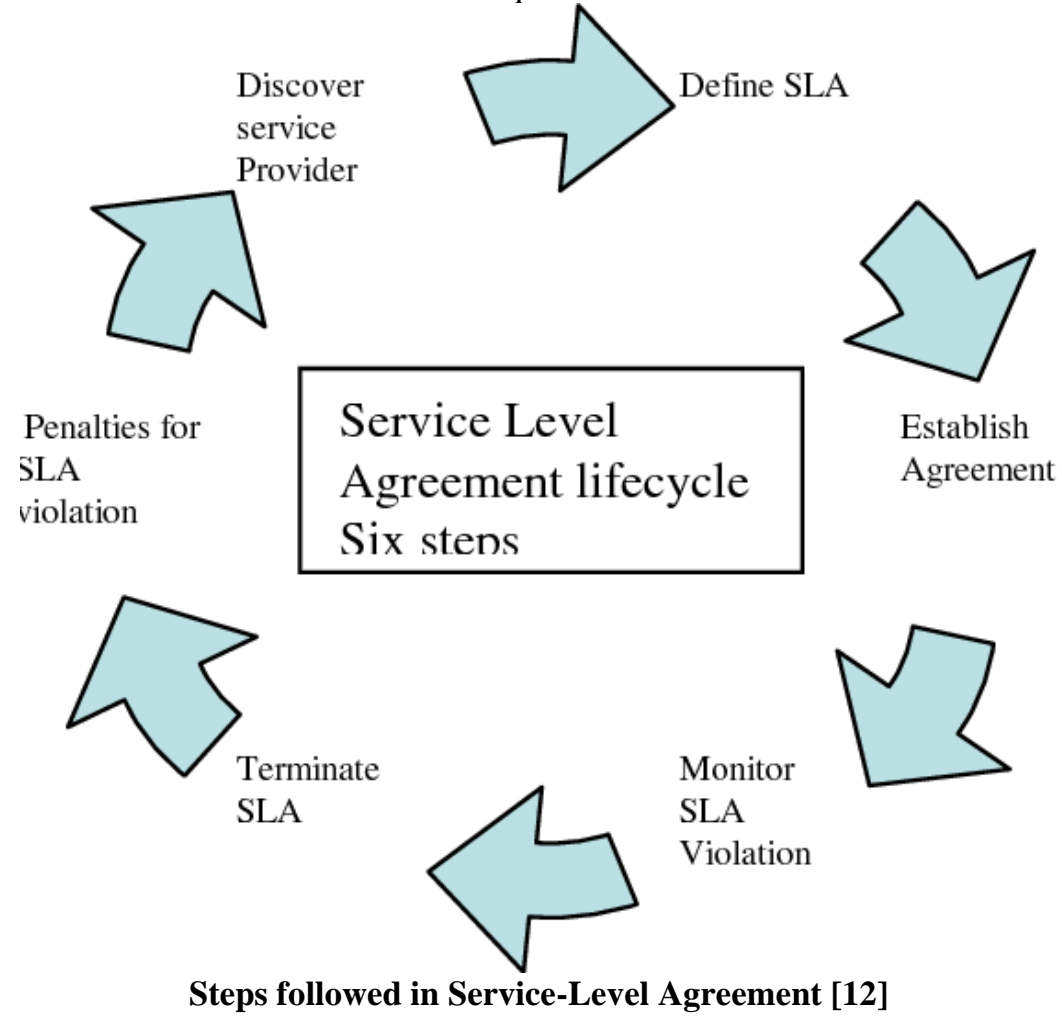

Quota, on the other side is used by a user which states that they should perform their actions within the limits of service provided from the provider side. It fixes the limit of the client using the services provided by the service providers. Cloud computing consist of many capabilities such as virtual machines, storage services, all are just a small part in the system. [13] Amazon alone, offers 150 different services of such kind in APIs. All of these different services work upon SLA. They couldn't go against the SLA setup and have to perform the work done under its structured program.

Let's talk about Cloud Providers [14], the two of the most important concepts in this is identity, and access management. IAM (identity and access management) is exactly opposite to Whoami. It acts as the front entry towards the structure and should be guarded properly in order to stop malicious subjects. The services which are provided to the user could be secured by applying various security policies containing security rules for different resources. Suppose any organization have " $n$ " number of employees, now it becomes really hard task to manage policies for each and every resource, hence roles are introduced to the system which assigns the different access level. Roles allows the group together into one single unit and used at any place in the cloud also, could be used outside the system if one is working with any third party or the consultant. 

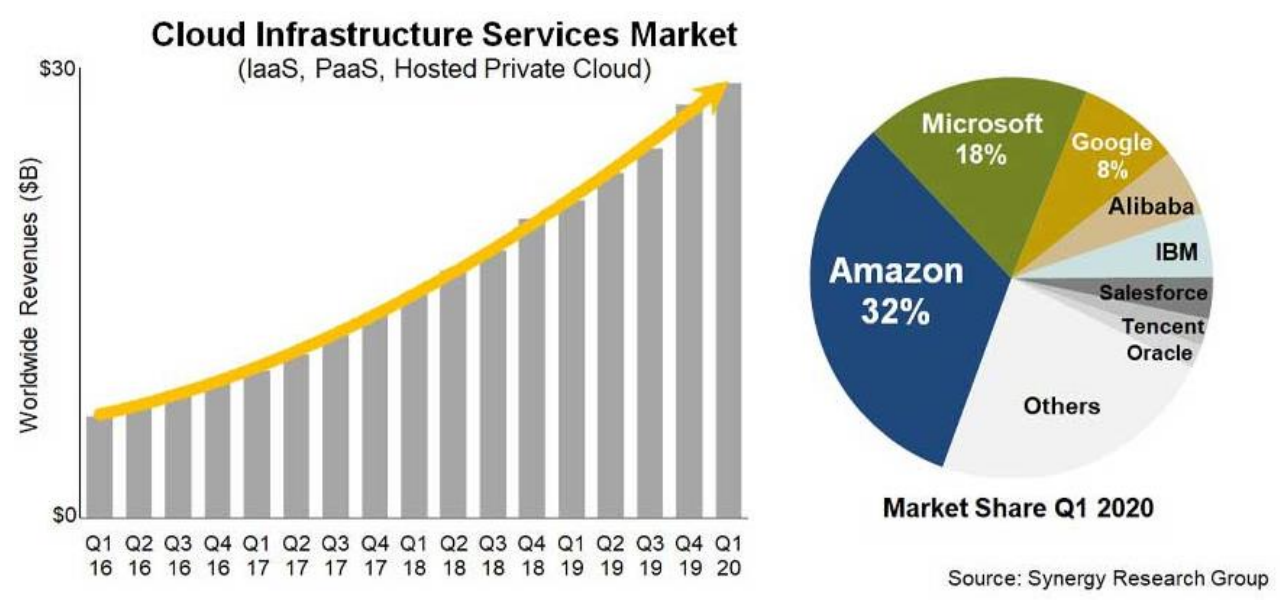

A chart of May 05,2020, after the pandemic "Covid-19" hit hard on the world. [15]

The interactions between various machines could be stated on a resource-based policy, or different service accounts. This allows any virtual machine to access the database if needed somewhere else in the cloud, providing machine to machine Permission.

Computing resources is the backbone of the cloud. Virtual machine could be created with the help of compute engine on Google cloud. While creating this machine, user would be presented with few options. In this, the Region provides the location of the data center where the data lies. It is a physical location in the cloud campus. The region lying close to the end user will provide the fastest services. The companies need to follow stated regulations regarding data centers, storing customer's data. If we look upon the scenario, each region acts as a campus for various isolated data centers. This helps when one has heavy workloads, these data or workloads could be put into one region, which in turn will be divided into multiple zones. (Isolated data centers). If due to any reason the crash occurs in one of the data centers, users still has the reliable service in that particular region providing higher redundancy and higher availability. From all over, the clouds have the highest data center situated across the world, with few secret government locations.

The other option available in this is "Machine Type". The amount of memory and the CPU is highly dependent on the type of machine a client chooses, cost is also dependent on the size of machine. The amount needs to be utilized by the user is for that particular instance, once the instance has been stopped, the cost of that particular used instance needs to be paid. Cloud computing highly relies on "pay-as-you-go" service. [16] This leads to opt the operating system by selecting the disk image (boot disk: Consisting of VM files and operating systems). User could choose from Linux or Windows, option is provided, specifying the services required by the user. If we talk about the security provided and this kind of scenario, the firewall is present in a default mode which prevents the invasion or connection with the estimated threats. It controls the traffic over the network.

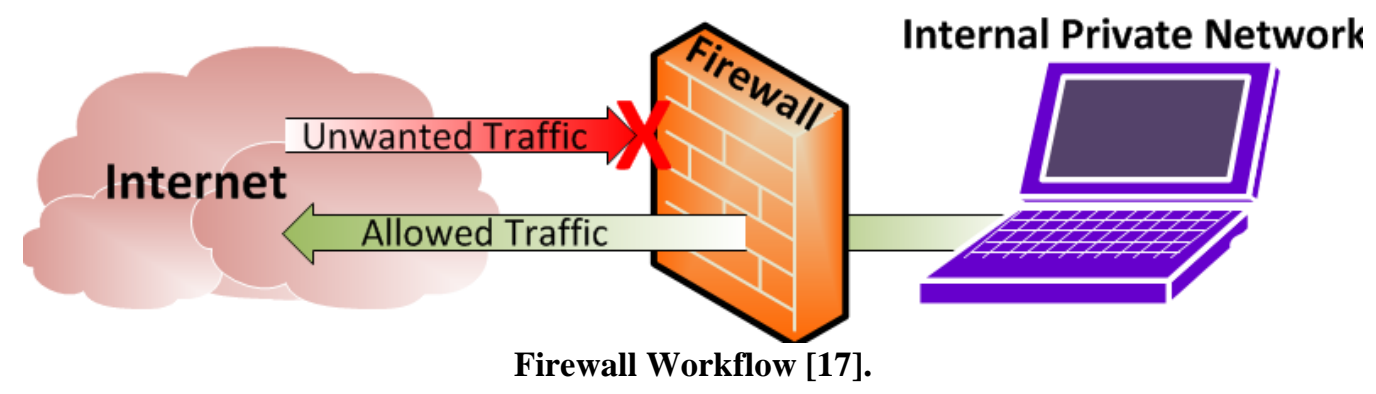

In networking we have an internal IP address as well as an external IP address both of these values are temporarily active during the instances, if any service resides on the IP address, one can always take a static IP address. The internal IP version is required to communicate with other compute instances over the same cloud. External IP version helps in communicating with the services residing outside that particular cloud.

Egress, the outbound data and Ingress, [18] the inbound data, also a part of networking. Egress helps in sending the data from the inside to the outside world, also should be taken more care as the data from outside world could affect directly on the cost. Ingress on the other hand is quite opposite, where data needs to be sent from outside world into their instance. 
For the stimulated computer we have been talking about, for the instance running on this system one has to access the command line, we can do that directly on the console with the cloud shell session (this is a command line present in a virtual mode). Inside the session one is free to operate it as the user wanted.

\section{VERTICAL SCALABILITY}

Many a times virtual machine is not even necessary, with compute resources one can handle the complete process. A large factor depends on its scalability or how we are scaling the system: suppose an app established on the virtual machine, there are "N" number of users which are occupying the CPU and the memory unit of this system, vertical scalability could be used here, scaling vertically makes the thing looks bigger. Adding more CPU, as well as memory unit makes the specific virtual machine more powerful as compare to the original virtual machine. As everything got its limit the same happens with vertical scalability.

\section{HORIZONTAL SCALABILITY}

The other scenario could be used as, instead of increasing the size of a virtual machine and making it bigger, one can be involved in making more virtual machines, created in all of the regions over the world and distributing the workload accordingly to the system over the different regions. Performing so, one needs to get concerned over the workload as distributed among the regions. [19]

Thus load balancing comes into the picture. There could be many chances of the complete traffic going to certain virtual machines and the other virtual machines are sitting idly for the longer period of time hence all the cloud system offers the load balancing service to helps manage the workload distributing evenly among the different machines.

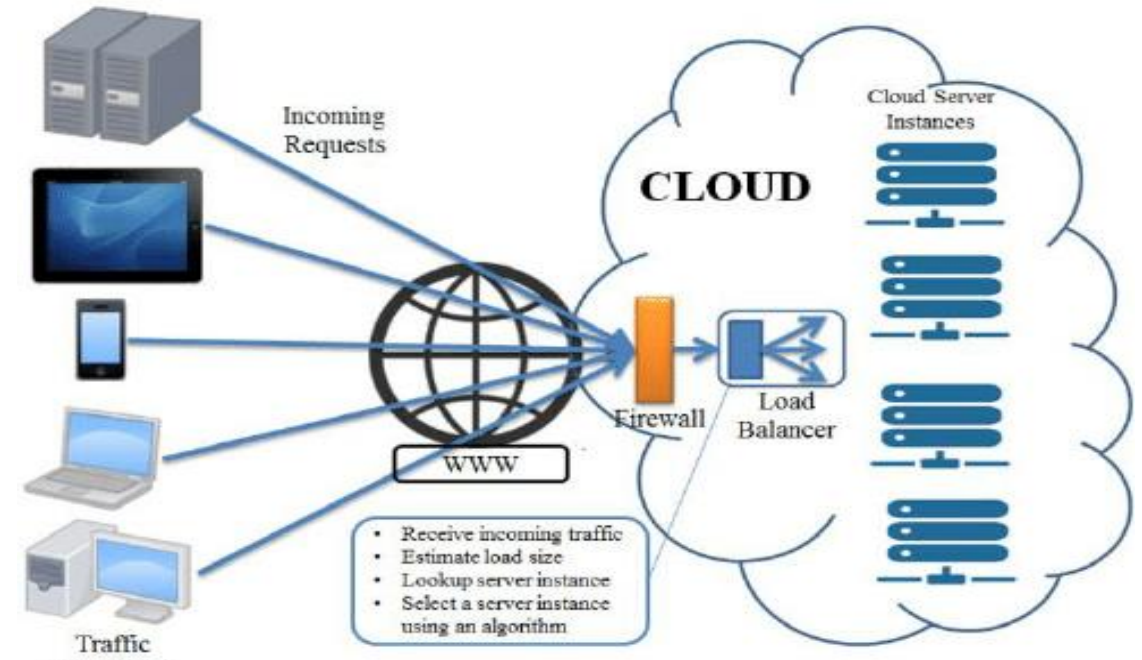

Load Balancing in Cloud [20]

Scaling the computer resources is not always the only option. There are two computing systems which does not require scaling the infrastructure:

\section{- $\quad$ Containerization [21]}

Provides a stimulated operating system. If we talk about a common virtual machine, it usually sits on the hardware of the system, but on the other hand, a container sits on top of operating system to stimulate another operating system or application provided. This development helps in putting any of your application in the container and deploying it to any other cloud, avoiding the scenario introduced called vendor lock-in. The cloud contains container registry (all the containers stored at a single place) in the system, hence any container could be uploaded to be used across various different services. Most of the organization, in today's world, contains multiple containers to run various services in the system such as one container could be used for sending email, another for algorithm, another for Web servers and so on.

\section{- Kubernetes}

The main work of $\mathrm{K} 8$ is to manage all the containers in a group of pods, scaling those pods up or down, according to the utilization of the traffic. Once the traffic goes up, K8 allows more containers and once the traffic is up to run in virtual machines it results in more virtual machines. As soon as the traffic goes of those virtual machines containers are taken off from the cloud atmosphere, hence the cost is saved, as the machines will be sitting idle on the system. 
Kubernetes Architecture

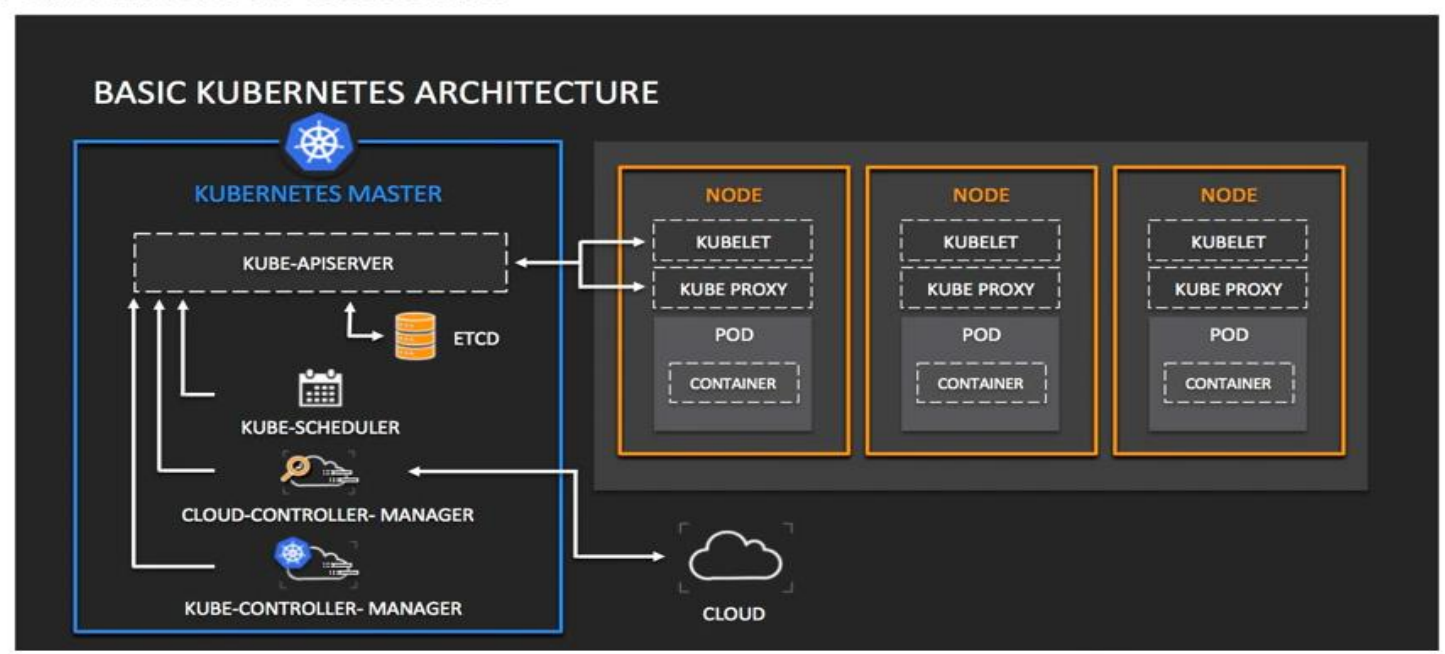

Working in $\mathrm{K8}$ atmosphere [22]

There is something called Serverless, introduced in 2014 with AWS lambda, here stating "functions-as-a -service", it helps in running code as well as responsible for the events generated, such as a general HTTP request or when something happens on any other cloud database. It scales automatically with providing a secure atmosphere on to a cheap price range. It also works on the concept of "pay-as-you-go" without worrying about any virtual machines, any containerization etc.

\section{U.S. serverless architecture market size, by service, 2015 - 2025 (USD Million)}

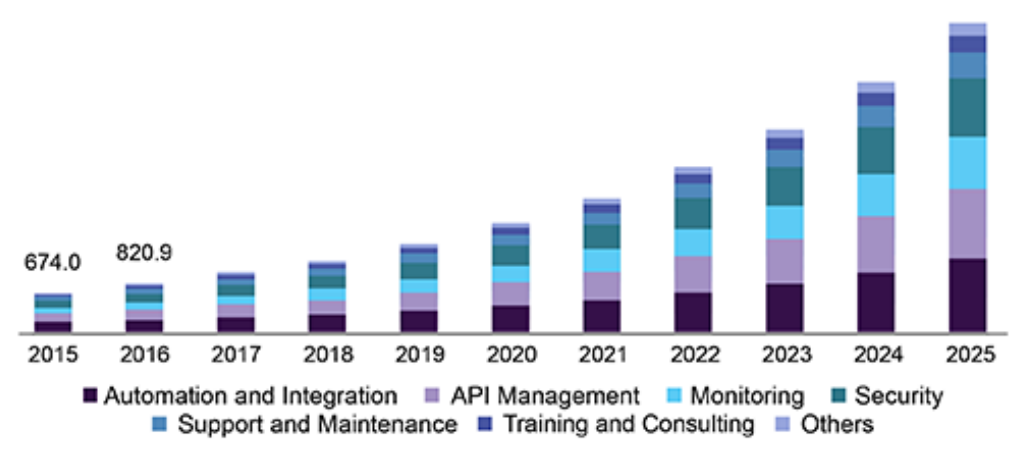

Server less Architecture Market analysis. [23]

\section{RESULT \& CONCLUSION:}

By October 23, 2019, Google announced to provide the world with Quantum Supremacy, this machine is hundred and million times faster than our 16 inches MacBook. One month later AWS released "Quantum computing-asa-service" for the research purpose.

- $\quad$ The market shares of AWS leading the market with run rate of $\$ 51$ billion is growing at $28 \%$, with Azure run rate of $\$ 29$ billion growing at $48 \%$ and Google cloud at third position growing with run rate of $\$ 15$ billion at $47 \%$ rate, calculated by the Synergy research group by February 01,2021. [24] 
Vol. 10, Issue 9, September 2021

DOI: 10.17148/IJARCCE.2021.10907

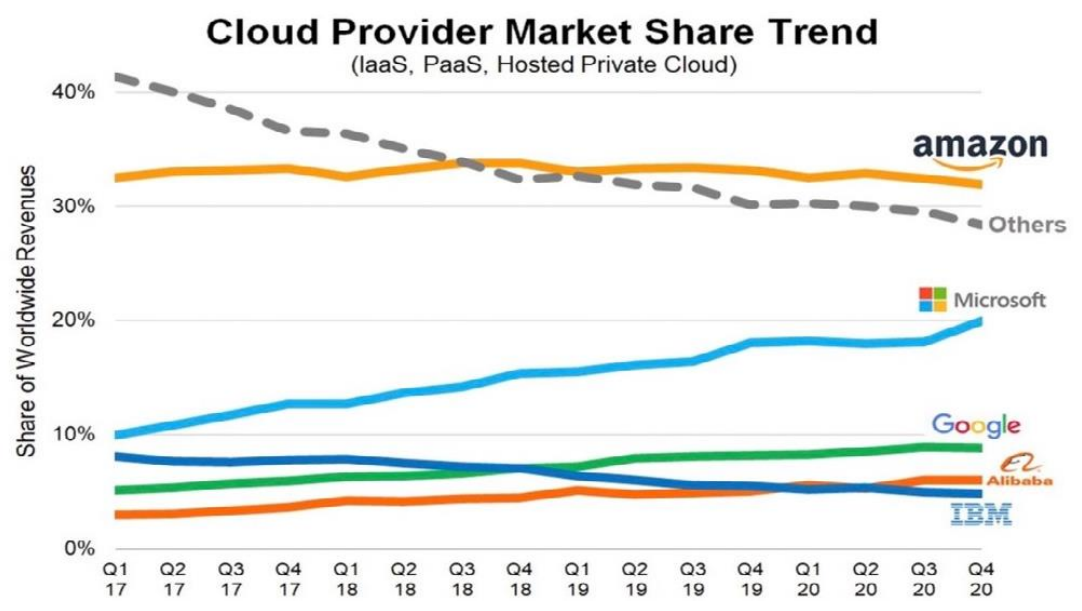

- $\quad 90 \%$ companies are on the cloud and more than $60 \%$ of the work load runs over it. Over $30 \%$ of the budget of IT is being applied to the cloud, leading billion dollar revenues, with increase in numbers, over the coming years.

- $\quad$ Highly optimized cloud campuses are situated nearby the rivers, for optimizing the cooling and also provided with a guaranteed up time to $99.99 \%$ and above.

- The earlier most significant use cases are helping us now to produce the new concept and scenarios over changing time period. In 2021, more businesses are attracted towards cloud model as it leads to various benefits over mobility over security, productivity, cost efficient, software updating.

- In today's world, artificial intelligence has highly increased the efficiency of cloud Computing. It helps in various skills in accessing machine learning functions (Image Recognition tools, knowledge processing, recommendation engines).

- $\quad$ Cloud believes in increasing the efficiency as well as productivity working with the enterprises of all sizes in all fields. New famous vehicles, smart flying infrastructure, pandemic rush response planning provides a new field for research.

- $\quad$ Machine plays a big part in cloud data centers and keep its running. The hardware and the power usage, in this expensive environment is managed by AI algorithms. The research in the field is continue to meet the new breakthroughs and impacts as well as data sciences efficiencies.

- $\quad$ Cloud provider, over the years made a lot of money with the companies providing them and the companies using them. Amazon Web Services alone makes $28 \%$ of its income from total sale. It's a win-win situation from both end as instead of buying managing the hardware, the user started to use the desired infrastructure or services which are available at fingertips, making it easier to scale things up and down and hence, no need of an IT developer to align the system.

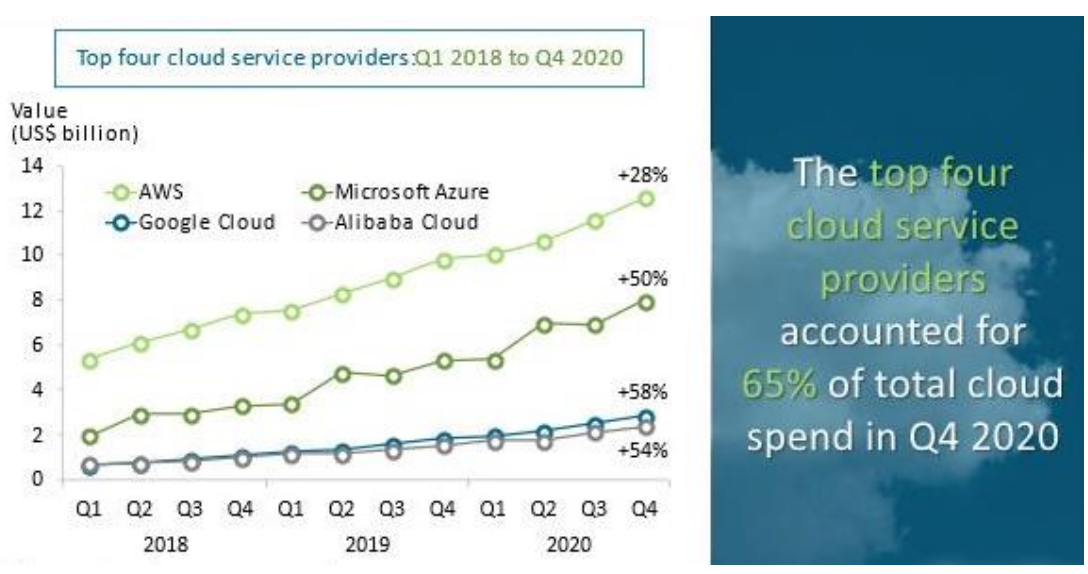

Top cloud service providers in 2021 by Canalys [25]

- $\quad$ Before 2019, user doesn't have a control over runtime, thus, installing a specific level of O.S was a difficult thing to do, as one doesn't have actual control over the runtime, in 2020 AWS releases the run time APIs as well as Google released a "Cloud Run" [26] service, hence making it possible to run a service in a serverless environment, turning out to be the easiest as well as cost-effective way. 


\section{International Journal of Advanced Research in Computer and Communication Engineering}

Vol. 10, Issue 9, September 2021

\section{DOI: 10.17148/IJARCCE.2021.10907}

According to Forrester [27], the global public market structure will grow up to $35 \%$ to $\$ 120$ billion in the year to 2021, after recovering from the pandemic. During the pandemic many companies shifted to cloud-based approach, as it was easier to handle as it could be operated from anywhere, online systems. It is assumed that there will be a reshuffling done between the top three cloud providers as in the revenue state both clouds i.e.: Alibaba cloud and the Google cloud are very close to each other.

- In 2020-2021, Server less is to be counted among the top five fastest growing Platform service cloud, according to reports produced by Flexera [28]. The applications in future will be developed in such a way so that they can take the advantages of server less model.

- $\quad$ The market value of cloud business process services, PaaS, SaaS, IaaS, security services is increasing day by day as a lot of companies are now accepting the cloud base, according to Gartner [29].

- $\quad$ Also according to Gartner, by the end of 2020, $95 \%$ of cloud security failures will be due to customer's fault or their lack of understanding and not the cloud providers fault.

- It is stated that by 2025 , cloud providers will sell solutions and not infrastructure. Although infrastructure will remain intact but more weightage will be given to the solutions.

- $\quad$ By this year only AWS, Azure, GCP Will continue to support all the primitive services. EC2, S3, message buffers (SQS), these powerful tools which are used by the developers nowadays will be providing with a common API having the same familiar construct.

There is one huge downside in providing the infrastructure by the cloud service provider, for the cloud providers, which is that the APIs will lead to a race to the bottom on Price. Suppose, if developer only uses the core components of the cloud platform, such as Storage, Compute, Queues, K8 functions, Databases, Serverless functions, providers have to compete on the features and the prices could go to a very low margin threshold. As customers are not bounded by any particular cloud, in future it will become more easy to hop to a different cloud if the other cloud providers offer discounts or reliability problem solutions for that particular instance.

Enterprises are struggling to get on to AWS as due to its massive engineering commitments, cloud providers will make the customers to hire expensive employees in future in order to use the platform.

- $\quad$ SaaS vendors will capture most of the actual values of the cloud. If a company migrates from On-premises on to a workday, the workday captures $95 \%$ of its value of running in the cloud.

- $\quad$ AWS, built all of the nuts and bolts which provides a giant cloud native SaaS tools.

- $\quad$ Cloud providers will continue to provide out-of-the-box solutions services such as big query/red shift [30], exposes the power of SQL to business analyst, soon snowflake will be out of the loop.

- $\quad$ AWS is building the tools for a new code or low code framework with AI Machine Learning tools.

- Google cloud provider is trying for a big play, with the concept of business analyst doing machine learning Directly from GCP console [31]. There will be no need for intermediary start-ups for Google's revenue.

- Google, Amazon and Microsoft will continue to build more apps, websites using the in-house tool. The usable version of current SaaS tools will start appearing as cloud services by 2024.

- $\quad 50 \%$ of the enterprises or the organizations will directly interact with AWS services, using AWS as an end-toend service.

\section{REFERENCES:}

[1] https://en.wikipedia.org/wiki/Cloud_computing.

[2]https://www.tutorialspoint.com/amazon_web_services/amazon_web_services_basic_architecture.htm

[3] Reseller Club, May 24,2019, “Type-1 and Type-2 hypervisor: what makes them different.”, https://medium.com/teamresellerclub/type-1-and-type2-hypervisors-what-makes-them-different-6a1755d6ae2c

[4] https://www.nakivo.com/blog/hyper-v-virtualbox-one-choose-infrastructure/

[5] https://fireship.io/lessons/cloud-computing-in-2020/

[6] Rafat Ara, Md. Abdur Rahim, Sujit Roy, Dr. Uzzal Kumar Prodhan, June 2020, "Cloud Computing Architecture, Services, Deployment models, Storage, Benefits Challenges.”, https://www.researchgate.net/publication/341788106_Cloud_Computing_Architecture_Services_Deployment_Models_Storage_Benefits_and_ Challenges

[7] https://www.researchgate.net/figure/Cloud-Computing-Architecture-and-Service-Models-11_fig1_318649453

[8] Gautam Raturi, August 05,2021, "Why should Business go for Backend-as-a-Service in 2021!", https://medium.com/an-idea/why-shouldbusinesses-go-for-backend-as-a-service-in-2021-57571737c0cf

[9] Ajay Sarangam, March 03,2021, “Types of cloud environment: a simple overview (2021)", https://www.jigsawacademy.com/blogs/cloudcomputing/cloud-environment/

[10] https://www.researchgate.net/figure/Cloud-computing-deployment-models-6_fig2_308919598

[11] https://www.cloudflare.com/en-in/learning/cloud/what-is-multicloud/

[12]

https://www.semanticscholar.org/paper/Service-Level-Agreement-(SLA)-for-Cloud-Computing-KimBarua/5ef313a98b54d4287dbff4045d77d298d8d0e32d 


\section{International Journal of Advanced Research in Computer and Communication Engineering}

Vol. 10, Issue 9, September 2021

DOI: $10.17148 / I J A R C C E .2021 .10907$

[13] Isaac Odun-Ayo, Blessing Udemezue, Abiodun Kilanko, January 2019, "Cloud Service-level Agreements and Resource Management.”, https://www.researchgate.net/publication/345468812_Cloud_Service_Level_Agreements_and_Resource_Management

[14] http://d1.awsstatic.com/analyst-reports/Report_CVU_CC_AWS_ENGL_final.pdf

[15] https://www.missioncriticalmagazine.com/articles/92949-covid-19-fails-to-slow-cloud-spending

[16] Jingyu Liu, Jing Wu, Linan Sun \& Hailong Zhu, June 15,2020, "Image data model Optimization method based on Cloud Computing.", https://journalofcloudcomputing.springeropen.com/articles/10.1186/s13677-020-00178-7

[17] https://www.tunnelsup.com/what-is-a-firewall/

[18] https://cloud.google.com/vpc-service-controls/docs/ingress-egress-rules

[19] Abid Nisar, Waheed Iqbal, Fawaz Bokhari, Faisal Bukhari, Khaled Almustafa, (PUCIT), University of Punjab, Lahore, Pakistan, "Dynamic Horizontal and Vertical Scaling for Multi-Tier Web application.”, https://www.techscience.com/iasc/v26n2/39949

[20] https://www.researchgate.net/figure/Load-Balancing-in-Cloud_fig1_274007923

[21] Adam Muc, Tomaz Muchowski, Albert Zawadzki, Adam Szelezinski, September 2020, “containerization of Server Services.", https://www.researchgate.net/publication/344031334_Containerization_of_Server_Services

[22] https://www.oracle.com/cloud-native/container-engine-kubernetes/what-is-kubernetes/

[23] https://www.grandviewresearch.com/industry-analysis/serverless-architecture-market

[24] https://www.telecomtv.com/content/digital-platforms-services/now-cloud-market-leader-aws-needs-a-new-chief-40772/

[25] https://www.c-sharpcorner.com/article/top-10-cloud-service-providers/

[26] Ekaba Bisong, March 21,2020, “Cloud Run: Google cloud Text to speech API.” , https://towardsdatascience.com/cloud-run-google-cloud-text-tospeech-api-dff308665c70

[27] https://www.techrepublic.com/article/top-cloud-trends-for-2021-forrester-predicts-spike-in-cloud-native-tech-public-cloud-and-more/

[28] https://www.flexera.com/about-us/press-center/flexera-releases-2021-state-of-the-cloud-report.html

[29] https://www.gartner.com/en/newsroom/press-releases/2020-11-17-gartner-forecasts-worldwide-public-cloud-end-user-spending-to-grow-18percent-in-2021

[30] https://www.xplenty.com/blog/redshift-vs-bigquery-comprehensive-guide/

[31] https://www.g2.com/products/google-cloud-console/reviews 